\title{
TmDOTP : An NMR- based Thermometer for Magic Angle Spinning NMR Experiments
}

\author{
Zhang, Dongyu ${ }^{\mathrm{a}}$, Itin, Boris ${ }^{\mathrm{b}}$ and McDermott, Ann E. . $^{\mathrm{a}^{*}}$ \\ aDepartment of Chemistry, Columbia University, New York, NY 10027; \\ ${ }^{b}$ New York Structural Biology Center, New York, NY, 10027; \\ *To whom correspondence should be addressed. Email: aem5@columbia.edu
}

Keywords: Nuclear magnetic resonance, Magic-angle spinning, Heating, Real-time NMR temperature measurement, Dielectric loss, TmDOTP5-

\begin{abstract}
Solid state NMR is a powerful tool to probe membrane protein structure and motions in native lipid structures. Sample heating, caused by magic angle spinning and radio frequency irradiation in solid state NMR, produces uncertainties in sample temperature and thermal broadening caused by temperature distributions, which can also lead to sample deterioration. To measure the sample temperature in real time, and to quantify thermal gradients and their dependence on radio frequency irradiation or spinning frequency, we use the chemical shift thermometer TmDOTP, a lanthanide complex. Compared to other NMR thermometers (e.g., the proton NMR signal of water), the proton spectrum of TmDOTP exhibits higher thermal sensitivity and resolution. In addition, the $\mathrm{H}_{6}$ proton in TmDOTP has a large chemical shift $(-175 \mathrm{ppm}$ at $275 \mathrm{~K})$ and is well resolved from the rest of the proton spectrum. We identified two populations of TmDOTP, with differing temperatures and dependency on the radio frequency irradiation power, within proteoliposome samples. We interpret these populations as arising from the supernatant and the pellet, which is sedimented from the sample spinning. Our results indicate that TmDOTP is an excellent internal standard for monitoring temperatures of biophysically relevant samples without distorting their properties.
\end{abstract}

\section{Introduction}

Magic angle spinning (MAS) solid-state nuclear magnetic resonance (SSNMR) is a powerful technique for studying biomolecules ${ }^{1}$, including: protein assemblies in near native conditions ${ }^{2} 1$, membrane proteins ${ }^{3-5}$ and amyloid fibrils ${ }^{6-8}$. SSNMR provides rich information on protein molecular structure and motions. Restricted global molecular motions in solids allow for the retention of dipolar couples, which enables direct measurement of distances and local orientations. Many of the most exciting developments in SSNMR however, involve pulse sequences with long and strong radio frequency (RF) irradiation elements; Sample heating from magic angle spinning and RF irradiation has been cause for concern ${ }^{9,10}$ Elevated and uncalibrated temperatures within the sample complicates the interpretation of dynamics and other properties. Moreover, heating gradients within the MAS rotor may contribute to peak broadening.

Sample heating originates in part from friction between bearing gas and the rotor during MAS ${ }^{10,11}$. Heat is also generated from RF irradiation during high power decoupling due to inductive dielectric heating on conductive or dipolar samples ${ }^{9,12,13}$. The application of high power oscillating electric field causes free charges and permanent electric dipoles to move, generating kinetic energy, which dissipates in the surrounding sample as heat ${ }^{14}$. The absorption of RF energy is maximized when $\omega \tau=1$, where $\omega$ represents the frequency of oscillating field and $\tau$ is the characteristic relaxation time of the molecule ${ }^{14,15}$. RF heating is of particular concern in SSNMR 
experiments on biological samples due to the resistive losses from the high concentration of ions in typical biological buffers and the dipolar losses from the presence of mobile permanent dipoles such as in hydrated lipids ${ }^{12,15-18}$. Since the heating mechanism is difficult to completely avoid for such samples, it is critical to monitor the temperature changes and heating gradient in order to control the sample temperature during SSNMR experiments.

Here, we use thulium 1,4,7,10-tetra-azacyclododecane-1,4,7,10-tetrakis (methylene phosphonate) TmDOTP $^{5-}$ (CAS: 30859-88-8), specifically the $\mathrm{H}_{6}$ proton chemical shift, as an internal thermometer to measure the temperatures for biological samples during SSNMR experiments (Figure 1A). TmDOTP ${ }^{5-}$ is a water soluble paramagnetic complex, which is known to have strongly temperature dependent chemical shifts for ${ }^{1} \mathrm{H},{ }^{13} \mathrm{C}$ and ${ }^{31} \mathrm{P}$ (Figure 1B). Compared with other compounds that have excellent thermal resolution in chemical shifts, such as $\mathrm{Pb}\left(\mathrm{NO}_{3}\right)_{2}$, $\mathrm{KBr}$ or $\mathrm{Sm}_{2} \mathrm{Sn}_{2} \mathrm{O}_{7}{ }^{19}$, $\mathrm{TmDOTP}^{5-}$ is convenient to measure, since temperature measurements are made in situ, without changing samples or probe tuning. The $\mathrm{H}_{6}$ proton was chosen for its moderately high temperature sensitivity and relatively narrower linewidth compared to the other five nonequivalent protons ${ }^{20}$. Due to its low toxicity, TmDOTP $^{5-}$ has been applied to clinical magnetic resonance to measure the temperature of tissue cells and tumor cells during surgery ${ }^{20}$.

A.

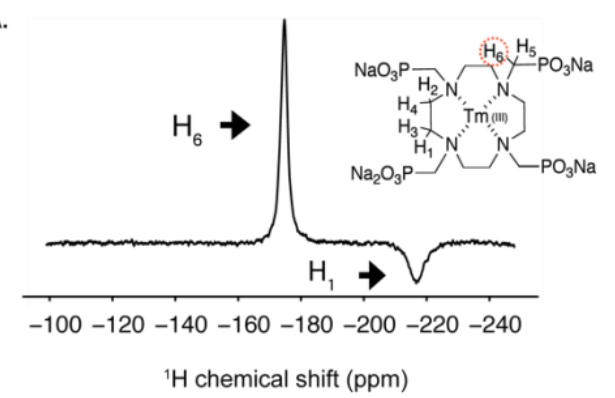

B.

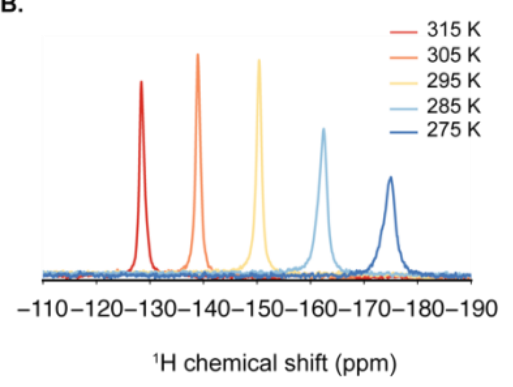

Figure 1. (A) Molecular structure of TmDOTP with $\mathrm{H}_{6}$ highlighted and portion of the ${ }^{1} \mathrm{H}$ NMR spectrum of $25 \mathrm{mM}$ TmDOTP. The sample contains KcsA proteoliposome and $25 \mathrm{mM}$ TmDOTP. $\mathrm{H}_{6}$ is at $-175 \mathrm{ppm}$ while $\mathrm{H}_{1}$ is $-217 \mathrm{ppm}$. (B) Overlay of the spectra of the $\mathrm{H}_{6}$ proton in TmDOTP acquired at various temperatures. All spectra were collected on $900 \mathrm{MHz}$ with MAS frequency at $5 \mathrm{kHz}$. Chemical shift was referenced to the TMS at $0 \mathrm{ppm}$.

\section{Experiment and Method}

\section{Sample preparation}

74 TmDOTP (Macrocyclics, Inc.) buffer was made with 25mM TmDOTP (molecular weight: 914.2 $\mathrm{g} / \mathrm{mol}$ ), $20 \mathrm{mM}$ MOPS and $100 \mathrm{mM} \mathrm{KCl}$ at $\mathrm{pH} 7.5$ in $99.96 \% \mathrm{D}_{2} \mathrm{O}$ (Sigma). 10mg wt-KcsA was overexpressed and reconstituted into 3:1 DOPE/DOPG (wt/wt) liposomes as described previously ${ }^{21}$. Then the proteolipsome sample was resuspended and incubated with TmDOTP buffer (same conditions as above) for 2 hours before packing into a regular-wall zirconia Bruker $3.2 \mathrm{~mm}$ rotor with a silicon spacer on the top.

\section{NMR Spectroscopy}

81 Experiments were carried out using a $3.2 \mathrm{~mm}$ standard-bore E-free probe and $1.3 \mathrm{~mm} \mathrm{HCN}$ probe 82 on a Bruker Avance II $900 \mathrm{MHz}$ spectrometer. The temperature was regulated with VT gas (flow 
rate $1070 \mathrm{~L} / \mathrm{hr}$ ) and a heater in the probe. The VT control unit was calibrated using the chemical shift difference between the $-\mathrm{CH}_{3}$ and $-\mathrm{OH}$ groups of methanol ${ }^{22}$. The temperature of the system was allowed to equilibrate for at least 15 minutes after each temperature change. The pulse sequence used to measure heating from RF irradiation is shown in Figure 2. $\tau_{1}$ represents the duration of the heating pulse, which resembles high power decoupling. Unless otherwise specified, $\tau_{1}$ was kept at $30 \mathrm{~ms}$ and the recycle delay was $1 \mathrm{~s} . \tau_{2}$ is the delay time to study cooling. Owing to the short $\mathrm{T}_{1}$ of the TmDOTP $\mathrm{H}_{6}$ proton $(\sim 800 \mu \mathrm{s})$, we kept $\tau_{2}$ at $5 \mathrm{~ms}$ to limit heat dissipation before acquisition. A short spin echo $\left(\tau_{3}=40 \mu \mathrm{s}\right)$ is added before acquisition to suppress TmDOTP $\mathrm{H}_{1}$ signal, which has a larger temperature slope $(\mathrm{ppm} / \mathrm{K})$ and could interfere with $\mathrm{H}_{6}$ signal at high temperature. The one-dimensional MAS spectra were acquired using 8 dummy scans and 512 scans.

94

95

The ${ }^{13} \mathrm{C}-{ }^{13} \mathrm{C}$ dipolar assisted rotational resonance (DARR ${ }^{23}$ ) experiments with $50 \mathrm{~ms}$ mixing time were performed on the same $900 \mathrm{MHz}$ spectrometer with a MAS rate of $16.666 \mathrm{kHz}$ and a set temperature of $267 \mathrm{~K}$. Proton decoupling with the SPINAL64 ${ }^{24}$ scheme at $90 \mathrm{kHz}$ was applied during acquisition. The recycle delay was $2.5 \mathrm{~s}$. The ${ }^{1} \mathrm{H}$ and ${ }^{13} \mathrm{C}$ Dual-Receiver DARR experiment was performed on $3.2 \mathrm{~mm}$ standard-bore E-free probe with Bruker Avance NEO spectrometer operating at $700 \mathrm{MHz}$. The MAS rate was $12.5 \mathrm{kHz}$ and VT gas flow was $2000 \mathrm{l} / \mathrm{h}$. SPINAL64 decoupling was applied at $\omega_{1} / 2 \pi=90 \mathrm{kHz}$ on the proton channel during acquisition (15 ms) and 102 the recycle delay was $2 \mathrm{~s}$.

103

104

105

106

107

108

109

110

111

112

113

114

115

116

117

118

119

120

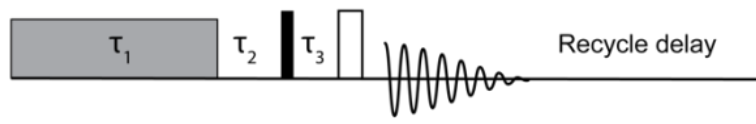

Figure 2. ${ }^{1} \mathrm{H}$ Pulse sequence used to measure the temperature increase from RF irradiation. The RF irradiation is applied for the duration of $\tau_{1} . \tau_{2}$ represents the delay before proton 90 pulse. A spin echo with $\tau_{3}=20 \mu \mathrm{s}$ is applied before acquisition to dephase the signal from $\mathrm{H}_{1}$ in TmDOTP

\section{Results and Discussion}

${ }^{1} \mathrm{H}$ NMR signals of $\mathrm{H}_{2} \mathrm{O}$ and TmDOTP as precise temperature measures

The water chemical shift is known to be sensitive to temperature and has been employed as an internal thermometer in several studies ${ }^{12,18,23}$. We compared the temperature dependence of the chemical shift of the $\mathrm{H}_{6}$ proton in TmDOTP versus the water proton in the same sample (Figure 3). The temperature dependency of the $\mathrm{H}_{6}$ proton in TmDOTP, $1.06 \pm 0.04 \mathrm{ppm} / \mathrm{K}$, is 2 orders of magnitude larger than that of water $\left(-1.1 \times 10^{-2} \pm 0.1 \times 10^{-2} \mathrm{ppm} / \mathrm{K}\right)$, while the full width at half maximum (FWHM), $(1.5 \pm 0.6 \mathrm{ppm})$, is one order of magnitude larger than that of water $(0.12 \pm 0.01$ $\mathrm{ppm})$. The uncertainty in the calculated temperature dependencies were dominated by the fitting error. Overall, TmDOTP allows for more accurate and precise temperature measurements than water. The homogeneous linewidth of the $\mathrm{H}_{6}$ proton calculated from $1 / \pi \mathrm{T}_{2}$ was about $980 \pm 60 \mathrm{~Hz}$ at $275 \mathrm{~K}$. The offset between homogeneous linewidth and the actual linewidth, $1.4 \mathrm{kHz}$, presumably is due to inhomogeneous broadening that cannot be refocused by a Hahn spin echo 


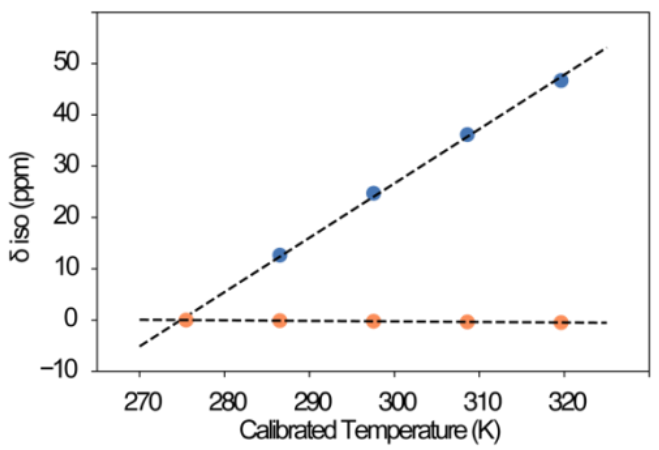

Figure 3. Comparison of the temperature dependency on the chemical shift of the $\mathrm{H}_{6}$ proton in TmDOTP (blue) and water proton (orange). $\Delta \delta_{\text {iso }}$ is the change in chemical shift relative to the shift at $275 \mathrm{~K}$. All spectra were collected with spinning frequency at $5 \mathrm{kHz}$ and gas flow rate at $1070 \mathrm{l} / \mathrm{h}$. The dashed lines represent linear least squares fitting to the data: $\delta i_{S O},_{\text {TIDOTP }}=1.06\left(\frac{p p m}{K}\right) T-291(\mathrm{ppm}) ; \delta$ iso $_{\text {,water }}=$ $-0.011\left(\frac{p p m}{K}\right) T+3.03(p p m)$. The error bars in both $\mathrm{x}$ and $\mathrm{y}$ dimensions for each data point are too small to be visualized. The error on slopes are dominated by the uncertainty from fitting. The ratio of slope and FWHM for TmDOTP and water are $0.7 \pm 0.3$ and $0.09 \pm 0.01$ respectively. The expansion of water chemical shift vs. calibrated temperature is shown in supplementary information (Figure S1).

Spinning heating scales with the rotor frequency

MAS induced sample heating was measured and fit in a $3.2 \mathrm{~mm}$ E-free (Figure 4) and a $1.3 \mathrm{~mm}$ probe (Figure S2). Samples with just TmDOTP buffer and KcsA proteoliposome were used to demonstrate the negligible dependency of the MAS heating on sample. The spinning frequencies and corresponding sample temperatures of TmDOTP were fit to second-order polynomial function according to previous studies: $\mathrm{T}=66 \frac{\mathrm{mK}}{\mathrm{Hz}^{2}} v_{r}^{2}-146 \frac{\mathrm{mK}}{\mathrm{Hz}} v_{r}+64 \mathrm{mK}$, where $=v_{r}=\omega_{r} / 2 \pi$ Notably, the $\mathrm{H}_{6}$ proton linewidth increased consistently with spinning frequency from $1636 \mathrm{~Hz}$ (at $2 \mathrm{kHz}$ MAS) to $3663 \mathrm{~Hz}$ (at $16 \mathrm{kHz}$ MAS). This increase inline width with MAS suggests that a heating gradient was caused by MAS. The line shape also grew more asymmetric with increasing MAS.

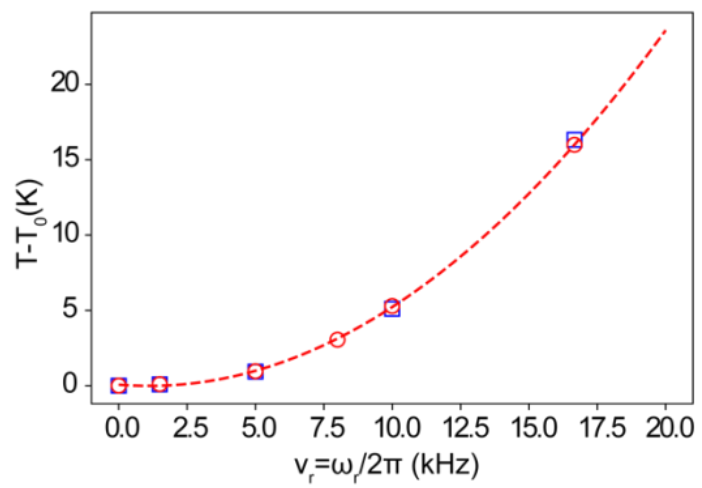

Figure 2. Sample temperature calculated from the $\mathrm{H}_{6}$ proton of TmDOTP as a function of spinning frequency. $\mathrm{T}_{0}$ is the temperature at zero spinning asymptote. TmDOTP buffer (red open circle) and KcsA proteoliposome samples (blue open square) were used here to demonstrate that MAS heating has little sample dependency. Data from the TmDOTP buffer were fit to second order polynomial function (red dash 
145 line): $T=66 \frac{\mathrm{mK}}{\mathrm{Hz}^{2}} v_{r}^{2}-146 \frac{\mathrm{mK}}{\mathrm{Hz}} v_{r}+64 \mathrm{mKT}$ Data for $1.3 \mathrm{~mm}$ probe is shown in supplementary 146 information (Figure S2).

147 The influence of TmDOTP on hydrated proteoliposome sample

148 We compared the KcsA proeoliposome spectrum with and without $25 \mathrm{mM} \mathrm{TmDOTP}$ and observed no significant changes in chemical shifts or overall spectral quality (Figure 5A). KcsA, a pH activated potassium channel from Streoptomyces lividans, is used here since the marker peaks of the protein are sensitive to $\mathrm{pH}$, temperature and potassium ion concentration changes ${ }^{3,21}$. This indicates that the paramagnetic nature of the TmDOTP complex has little to no effect on the properties of biological samples at the concentrations used. Marker peaks that belongs to the 155

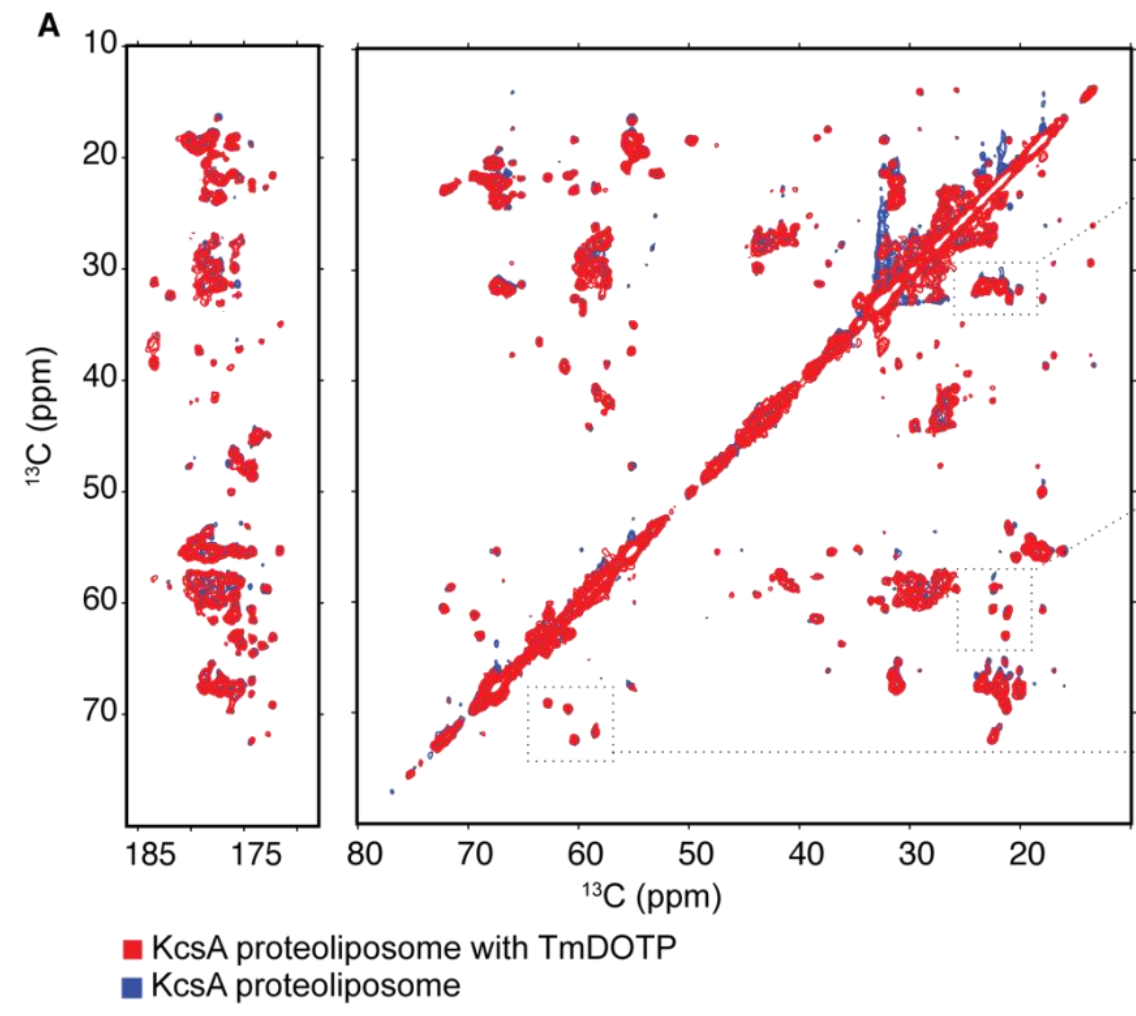

Figure 3. The effect of $25 \mathrm{mM}$ TmDOTP on KcsA proteoliposome. (A) Overlay of $2 \mathrm{D}{ }^{13} \mathrm{C}-{ }^{13} \mathrm{C}$ correlation spectra of KcsA (blue) and KcsA with 25Mm TmDOTP (red) acquired in DOPE/DOPG (3:1) liposome at $\mathrm{pH} 7.5$. The regions of KcsA selectivity filter marker peaks are highlighted and shown in (B) V76 C $\beta-\mathrm{C} \gamma$ (C) T74 $\mathrm{C} \alpha-\mathrm{C} \gamma$ and $\mathrm{T} 75 \mathrm{C} \alpha-\mathrm{C} \gamma$ (D) T74 $\mathrm{C} \beta-\mathrm{C} \alpha$ and $\mathrm{T} 75 \mathrm{C} \beta-\mathrm{C} \alpha$. The data suggest no significant changes in protein structure and conformation state with the addition of $25 \mathrm{mM}$ TmDOTP.

$\underline{\mathrm{RF}}$ heating is linear with pulse power, pulse length and duty cycle

To examine the heating of a biological sample during RF irradiation, the pulse sequence shown in Figure 2 was applied to a KcsA proteoliposome sample and the temperature was monitored using the chemical shift of the $\mathrm{H}_{6}$ proton in TmDOTP. The target temperature was set at $275 \mathrm{~K}$ on the VT control and the gas flow rate was $1070 \mathrm{~L} / \mathrm{hr}$. Continuous wave $(\mathrm{CW})$ irradiation that resembles proton heteronuclear decoupling was applied here and the RF power, pulse duration $\left(\tau_{1}\right)$, and duty 
cycle were varied respectively. $\tau_{2}$ was kept small $(5 \mathrm{~ms})$ here to limit sample cooling before acquisition. Figure 6 shows that heating is proportional to the RF power, duration of the pulse and duty cycle as discussed in the literatures ${ }^{12,14}$.

A

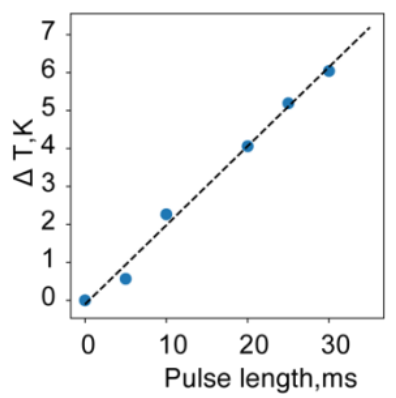

B

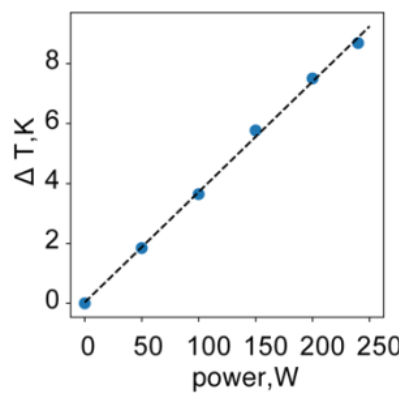

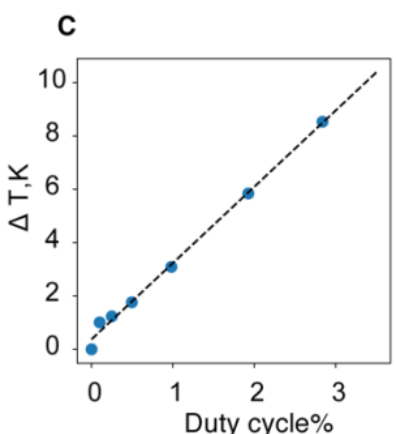

172

173

174

175

176

177

178

179

180

181

182

183

184

185

186

187

188

189

190

191

192

193

194

195

196

197

198

199

200

Figure 4. Plot of temperature increase calculated from TmDOTP chemical shift vs. (A) RF pulse length (field strength $=91 \mathrm{kHz}$, duty cycle $=2.8 \%)$. Data were fit using linear least square analysis $\left(\Delta \mathrm{T}=0.208 \tau_{1^{-}}\right.$ $0.105)$. (B) $\mathrm{CW}$ power amplitude $\left(\tau_{1}=30 \mathrm{~ms}\right.$ and duty cycle $\left.=2.8 \%\right)$. Data were fit using linear least square function $(\Delta \mathrm{T}=0.037 \mathrm{P}+0.032)$, $\mathrm{P}$ stand for RF power $(\mathrm{C})$ Duty cycle\% (field strength $=91 \mathrm{kHz}$ and $\left.\tau_{1}=30 \mathrm{~ms}\right)$. Data were fit using linear least square analysis $(\Delta \mathrm{T}=2.860 \mathrm{D}+0.375)$. $\mathrm{D}$ stands for duty cycle\%. All data were collected on a KcsA proteoliposome sample with $20 \mathrm{mM}$ TmDOTP on $3.2 \mathrm{~mm}$ Efree prob at $900 \mathrm{MHz}$. The spinning frequency was $5 \mathrm{kHz}$ and the target temperature was set at $275 \mathrm{~K}$.

\section{Inequivalent RF heating on pellet vs. supernatant}

One surprising finding from our RF irradiation study on the proteoliposome KcsA sample is that the $\mathrm{H}_{6}$ proton in TmDOTP peak splits into two components (denoted by peak 1 and peak 2) under $\mathrm{RF}$ irradiation on $3.2 \mathrm{~mm}$ E-free probe (Figure 7). The appearance of peak 2, which has a larger heating slope, only appeared under RF heating conditions, but not MAS (Figure S3). Moreover, the temperature reported by peak 2 matches with the one calculated from water proton chemical shift in the sample (Figure S4). However, the two distinct temperature populations are not resolved in water proton peaks possibly due to the broad linewidth $(130 \mathrm{~Hz})$. Therefore, we assigned the two peaks to TmDOTP in the pellet (peak 1) that sediments to the inner rotor wall due to the centrifugal forces generated by MAS and the peak 2 to the TmDOTP remaining in the center supernatant based on the agreement with the bulk water temperature measurement. The homogeneous linewidth calculated from $\mathrm{T}_{2}$ for the peak 1 and peak 2 are $815 \pm 39 \mathrm{~Hz}$ and $598 \pm 22$ $\mathrm{Hz}$ respectively. The divergent temperatures indicated by TmDOTP peaks might arise from different cooling speed along the radial axis of the rotor and the distinct heat capacity of water and proteoliposome. The data collected on a $1.3 \mathrm{~mm}$ solenoid probe is shown in Figure S5. At the same field strength, the uppermost temperature of the sample is consistently higher on $1.3 \mathrm{~mm}$ probe than $3.2 \mathrm{~mm}$ E-free probe and the heating gradient is continuous rather than peak splitting. The determined heating gradient can be as large as $18 \mathrm{~K}$ at the field strength of $90 \mathrm{kHz}$ using the 1.3 $\mathrm{mm}$ probe. The difference in line shape between $3.2 \mathrm{~mm}$ E-Free and $1.3 \mathrm{~mm}$ solenoid probes is due to the probe design and heating/cooling mechanism. 
A

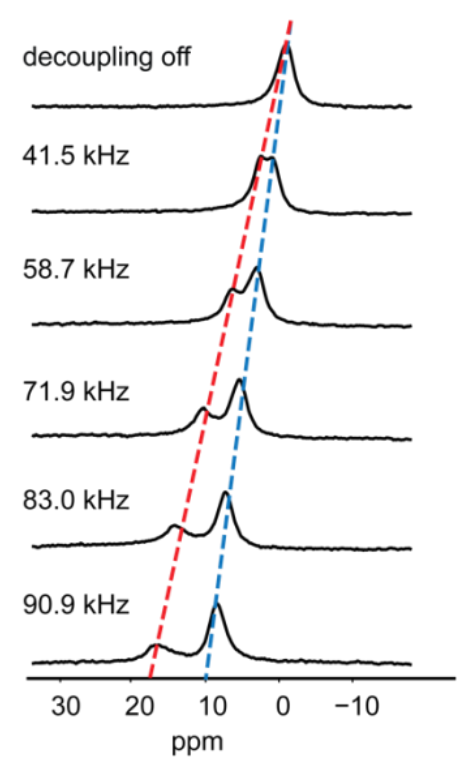

B

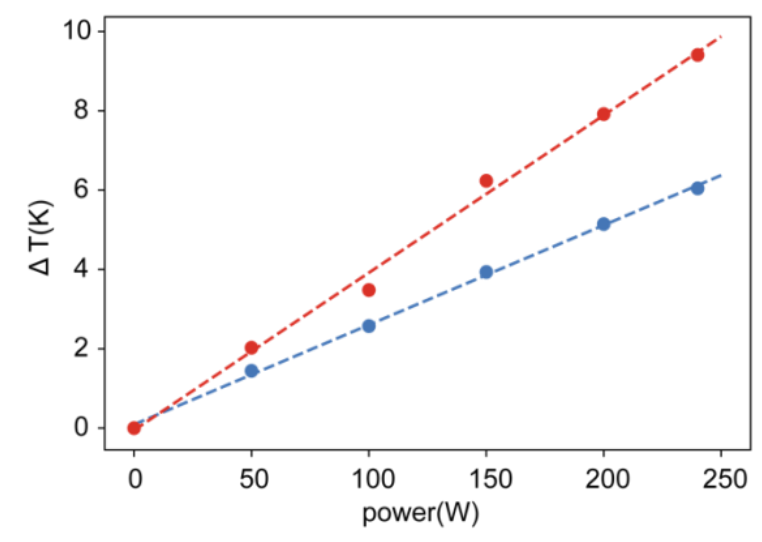

- peak 1 (right)

- peak 2 (left)

Figure 7. (A) The $\mathrm{H}_{6}$ NMR spectra of the proteoliposome sample with $20 \mathrm{mM}$ TmDOTP at neutral $\mathrm{pH}$ during different RF frequencies. Target temperature was set to $275 \mathrm{~K}$. The chemical shift of $\mathrm{H}_{6}$ was set to $0 \mathrm{ppm}$ when the decoupling pulse was off. This adjustment is for the convenience in temperature reading, since TmDOTP has a slope of nearly $1 \mathrm{ppm} / \mathrm{K}$. (B) Plot of RF power vs. sample temperature changes reported by the peak 1 and peak 2 from TmDOTP proton chemical shifts. All the data were collected on the $3.2 \mathrm{~mm}$ E-free probe at $900 \mathrm{MHz}$.

\section{Application and significance}

Owing to the fast relaxation rate and minimal perturbation on biological sample properties, TmDOTP can be incorporated into SSNMR samples to monitor real time temperature throughout an experiment. This may be crucial for samples and measurements that are sensitive to temperature changes, such as $\mathrm{R}_{1}{ }^{26}$. Here, we demonstrate the temperature mapping of a ${ }^{13} \mathrm{C}-{ }^{13} \mathrm{C}$ dipolar assisted rotational resonance (DARR) experiment using $20 \mathrm{mM}$ TmDOTP in KcsA proteoliposome sample. The experiment was carried out at Bruker $700 \mathrm{MHz}$ equipped with a 3.2 $\mathrm{mm}$ E-Free probe under $12.5 \mathrm{kHz}$ MAS. The multi-receiver feature on AVANCE NEO enabled an immediate $\mathrm{H}_{6}$ chemical shift measurement following every carbon acquisition. Figure $\mathrm{S} 6$ shows the temperature of KcsA sample increased about $0.5 \mathrm{~K}$ through the experiment caused by proton high power $(90 \mathrm{kHz})$ proton decoupling during the increasing evolution time t1. In addition, our data demonstrate that the heating from MAS and RF radiation are not additive. In order to obtain the precise temperature during an experiment, it is necessary to include a real time thermometer, such as TmDOTP, rather than simple extrapolation (Figure S7).

\section{Conclusions}

With a linear temperature dependency and large thermal resolution, we demonstrate that TmDOTP is an excellent internal thermometer for solid state NMR experiments on biological samples. The distinct proton chemical shift and short $\mathrm{T}_{1}$ enable an instant reading of the precise temperature in a biological sample. Comparing with common thermometer molecules that employed in SSNMR, such as ${ }^{207} \mathrm{~Pb}$ in $\mathrm{Pb}\left(\mathrm{NO}_{3}\right)_{2}{ }^{119} \mathrm{Sn}$ in $\mathrm{Sm}_{2} \mathrm{Sn}_{2} \mathrm{O}_{7}$, and $\mathrm{KBr}$, TmDOTP stands out in its low toxicity and nearly negligible perturbations on sample properties. Moreover, the 
proton detection eliminates the change in probe configuration and enables real time temperature reading and heat distribution measurement throughout an experiment. In addition, we observed two discontinuous temperature population in KcsA proteoliposome sample induced by RF irradiation. The two peaks were assigned to the pellet at the inner rotor wall and the center supernatant that result from MAS centrifugation. Finally, the discrepancy between the real temperature of decoupling while spinning and the extrapolated value from MAS and RF heating fitting curve shows the necessity and importance of obtaining real time temperature.

\section{Acknowledgments}

The NMR data was collected at the New York Structural Biology Center (NYSBC) with support from the Center on Macromolecular Dynamics by NMR Spectroscopy (CoMD/NMR) a Biomedical Technology Research Resource (BTRR) supported by U.S. National Institutes of Health (NIH) through grant number: P41 GM118302. The NYSBC is also enabled by a grant from the Empire State Division of Science Technology and Innovation and Office of Research Infrastructure Programs/NIH Facility Improvement Grant CO6RR015495. A.E.M. is a member of the NYSBC. This work was supported by NIH Grant R01 GM088724 (to A.E.M).

\section{Reference}

1. McDermott, A. Structure and dynamics of membrane proteins by magic angle spinning solid-state NMR. Annu. Rev. Biophys. 38, 385-403 (2009).

2. Gupta, S. \& Tycko, R. Segmental isotopic labeling of HIV-1 capsid protein assemblies for solid state NMR. J. Biomol. NMR 70, 103-114 (2018).

3. Wylie, B. J., Bhate, M. P. \& McDermott, A. E. Transmembrane allosteric coupling of the gates in a potassium channel. Proc. Natl. Acad. Sci. 111, 185-90 (2014).

4. Zhao, Y. et al. Gating Mechanism of Aquaporin Z in Synthetic Bilayers and Native Membranes Revealed by Solid-State NMR Spectroscopy. J. Am. Chem. Soc. 140, 78857895 (2018).

5. Liao, S. Y., Fritzsching, K. J. \& Hong, M. Conformational analysis of the full-length M2 protein of the influenza A virus using solid-state NMR. 22, 1623-1638 (2013).

6. Barclay, A. M., Dhavale, D. D., Courtney, J. M., Kotzbauer, P. T. \& Rienstra, C. M. Resonance assignments of an $\alpha$-synuclein fibril prepared in Tris buffer at moderate ionic strength. Biomol. NMR Assign. 12, 195-199 (2018).

7. Jaroniec, C. P. et al. High-resolution molecular structure of a peptide in an amyloid fibril determined by magic angle spinning NMR spectroscopy. Proc. Natl. Acad. Sci. 101, 711716 (2004).

8. Mompeán, M. et al. The Structure of the Necrosome RIPK1-RIPK3 Core, a Human Hetero-Amyloid Signaling Complex. Cell 173, 1244-1253.e10 (2018). 
9. D’Espinose De Lacaillerie, J. B., Jarry, B., Pascui, O. \& Reichert, D. 'cooking the sample': Radiofrequency induced heating during solid-state NMR experiments. Solid State Nucl. Magn. Reson. 28, 225-232 (2005).

10. Brus, J. Heating of samples induced by fast magic-angle spinning. Solid State Nucl. Magn. Reson. 16, 151-160 (2000).

11. Grimmer, A. R., Kretschmer, A. \& Cajipe, V. B. Influence of magic angle spinning on sample temperature. Magn. Reson. Chem. 35, 86-90 (1997).

12. Dvinskikh, S. V., Castro, V. \& Sandström, D. Heating caused by radiofrequency irradiation and sample rotation in 13C magic angle spinning NMR studies of lipid membranes. Magn. Reson. Chem. 42, 875-881 (2004).

13. McNair, D. S. Heat transfer in NMR of conductive samples with radiofrequency

14. Led, J. J. \& Petersen, S. B. Heating effects in carbon-13 NMR spectroscopy on aqueous (1978).

15. Li, C. et al. Analysis of RF heating and sample stability in aligned static solid-state NMR spectroscopy. J. Magn. Reson. 180, 51-57 (2006).

16. d'Espinose de Lacaillerie, J.-B., Jarry, B., Pascui, O. \& Reichert, D. “Cooking the sample": Radiofrequency induced heating during solid-state NMR experiments. Solid State Nucl. Magn. Reson. 28, 225-232 (2005).

17. Fowler, D. J., Harris, M. J. \& Thompson, L. K. Heat management strategies for solid-state NMR of functional proteins. J. Magn. Reson. 222, 112-118 (2012).

18. Wang, J., Zhang, Z., Zhao, W., Wang, L. \& Yang, J. Heating and temperature gradients of lipid bilayer samples induced by RF irradiation in MAS solid-state NMR experiments. Magn. Reson. Chem. 54, 753-759 (2016).

19. Fowler, D. J., Harris, M. J. \& Thompson, L. K. NIH Public Access. 112-118 (2013).

22. Ammann, C., Meier, P. \& Merbach, A. A simple multinuclear NMR thermometer. $J$. 
24. Fung, B. M., Khitrin, A. K. \& Ermolaev, K. An Improved Broadband Decoupling Sequence for Liquid Crystals and Solids. 101, 97-101 (2000).

309 25. Linser, R., Chevelkov, V., Diehl, A. \& Reif, B. Sensitivity enhancement using paramagnetic relaxation in MAS solid-state NMR of perdeuterated proteins. J. Magn. Reson. 189, 209-216 (2007).

312 26. Quinn, C. M. \& Mcdermott, A. E. Quantifying conformational dynamics using solid-state 313 R $1 \rho$ experiments. J Magn Reson 222, 1-7 (2012).

314 